Pacific

Journal of

Mathematics

FIBERWISE DIVERGENT ORBITS OF PROJECTIVE FLOWS WITH EXACTLY TWO MINIMAL SETS

HIROMICHI NAKAYAMA

Volume $229 \quad$ No. 2

February 2007 


\title{
FIBERWISE DIVERGENT ORBITS OF PROJECTIVE FLOWS WITH EXACTLY TWO MINIMAL SETS
}

\author{
HIROMICHI NAKAYAMA
}

\begin{abstract}
Let $\varphi_{t}$ be a nonsingular flow on a 3-dimensional manifold $M$. Denote by $\pi_{P}: P X \rightarrow M$ the projectivized bundle of the quotient bundle of $T M$ by the line bundle tangent to $\varphi_{t}$. The derivative of $\varphi_{t}$ induces a flow $\psi_{t}$ on $P X$, called the projective flow of $\varphi_{t}$. In this paper, we consider the dynamical properties of $\psi_{t}$ restricted to $\pi_{P}^{-1}(\mathfrak{M})$ for a minimal set $\mathfrak{M}$ of $\varphi_{t}$, under the condition that the restriction of $\psi_{t}$ to $\pi_{P}^{-1}(\mathfrak{M})$ has exactly two minimal sets $\mathfrak{N}_{1}$ and $\mathfrak{N}_{2}$. If $\varphi_{t}$ has no dominated splitting over $\mathfrak{M}$, we find two types of orbits of $\psi_{t}$ in the domain between $\mathfrak{N}_{1}$ and $\mathfrak{N}_{2}$ : one is "bounded below" and the other is "bounded above". As an application we prove that, if $\varphi_{t}$ is further assumed to be almost periodic on the minimal set, there is a dense orbit in that domain.
\end{abstract}

\section{Introduction}

Let $M$ be a closed oriented 3-dimensional manifold, and $X$ a nonsingular $C^{1}$ vector field of $M$. We denote by $T M$ the tangent bundle of $M$, and by $\varphi_{t}$ the flow generated by $X$. Let $\pi_{N}: N X \rightarrow M$ denote the quotient bundle of $T M$ by the 1dimensional bundle tangent to $X$. We take the projectivized bundle $\pi_{P}: P X \rightarrow M$ of $N X$, that is,

$$
P X=\bigcup_{z \in M}\left(\left(\pi_{N}^{-1}(z)-0\right) / v \sim k v\right)
$$

for $v \in \pi_{N}^{-1}(z)-0, k \in \mathbb{R}-0$. The derivative $D \varphi_{t}$ of $\varphi_{t}$ induces a projective flow $\psi_{t}$ on $P X$ (also called an inductance flow). Here, each fiber $\pi_{P}^{-1}(z)$ is oriented by the orientations of $T M$ and $X$, and $\psi_{t}$ preserves the orientation of fibers. Let $O(z, s)$ denote the orbit of $\psi_{t}$ passing through $(z, s) \in P X$, and let $O_{+}(z, s)$ and $O_{-}(z, s)$ denote the positive and negative orbits of $\psi_{t}$ passing through $(z, s) \in P X$.

In order to consider the dynamical properties of $\psi_{t}$, we will frequently use the renormalized linear Poincaré flow $v_{t}$, defined as follows: Let $|\cdot|$ denote the norm

MSC2000: 37B05, 37D30.

Keywords: minimal set, projective flow, dominated splitting.

Partially supported by Grant-in-Aid for Scientific Research (No. 15540078), Japan Society for the Promotion of Science, Japan. 
on each fiber of $N X$ induced from a Riemannian metric of $M$. We take an orthonormal basis $\left(v_{1}(z), v_{2}(z)\right)$ of $\pi_{N}^{-1}(z)$ for any point $z$ of $M$, with $\left(v_{1}(z), v_{2}(z)\right)$ not necessarily continuous with respect to $z$. The derivative $\left.D \varphi_{t}\right|_{\pi_{N}^{-1}(z)}$ is represented by a matrix, and hence we can define its determinant $\operatorname{det}\left(\left.D \varphi_{t}\right|_{\pi_{N}^{-1}(z)}\right)$. Since $\operatorname{det}\left(\left.D \varphi_{t}\right|_{\pi_{N}^{-1}(z)}\right)$ is independent of the choice of the orthonormal basis, we can define a new flow $v_{t}$ on $N X$ by

$$
v_{t}\left(z,[v]_{N}\right)=\left(\varphi_{t}(z),\left(\operatorname{det}\left(\left.D \varphi_{t}\right|_{\pi_{N}^{-1}(z)}\right)\right)^{-1 / 2}\left[\left(D \varphi_{t}\right)_{z}(v)\right]_{N}\right),
$$

where $[v]_{N}$ denotes the element of $\pi_{N}^{-1}(z)$ represented by the vector $v$ of $T_{z} M$. The flow $v_{t}$ preserves the area form on each fiber, and the next diagram commutes:

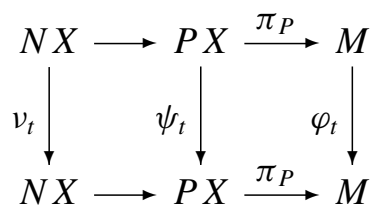

Let $\mathfrak{M}$ be a minimal set of $\varphi_{t}$ (that is, a closed $\varphi_{t}$-invariant set which is minimal with respect to the inclusion), and let $\widetilde{\mathfrak{M}}$ denote its lift $\pi_{P}{ }^{-1}(\mathfrak{M})$. Since the restriction of $\psi_{t}$ to $\tilde{\mathfrak{M}}$ is a flow on a compact set, $\psi_{t} \mid \widetilde{\mathfrak{M}}$ has a minimal set $\mathfrak{N}$. The set $\pi_{P}(\mathfrak{N})$ coincides with $\mathfrak{M}$ itself.

The number of minimal sets of $\psi_{t} \mid \widetilde{\mathfrak{M}}$ is essential for the study of the dynamical structure of $\psi_{t} \mid \widetilde{\mathfrak{M}}$. For example, if $\psi_{t} \mid \widetilde{\mathfrak{M}}$ has more than two minimal sets, it was already proved in [Nakayama and Noda 2005] that $\left.\varphi_{t}\right|_{\mathfrak{M}}$ is uniformly quasiconformal. In particular, if we further assume that $\varphi_{t}$ is a $C^{\infty}$ minimal flow on the 3-manifold $M$, then $M$ is the 3-dimensional torus $T^{3}$ and $\varphi_{t}$ is topologically equivalent to an irrational flow, by [Sullivan 1981; Brunella 1996; Ghys 1996] (see also [Matsumoto and Nakayama 1997]).

On the other hand, if $\left.\varphi_{t}\right|_{\mathfrak{M}}$ is uniformly quasiconformal, then $\psi_{t} \mid \widetilde{\mathfrak{M}}$ does not have exactly two minimal sets, because $\left.\varphi_{t}\right|_{\mathfrak{M}}$ is transversely conformal, again by [Sullivan 1981, p. 468], and a minimal set of $\psi_{t} \mid \widetilde{\mathfrak{M}}$ is still a minimal set after the constant rotation along the fiber with respect to this transverse conformal structure. (This can also be proved in the following way: if $\left.\varphi_{t}\right|_{\mathfrak{M}}$ is uniformly quasiconformal and the whole $\tilde{\mathfrak{M}}$ is not a minimal set of $\psi_{t} \mid \widetilde{\mathfrak{M}}$, we can show that, for any minimal set $\mathfrak{N}$ of $\psi_{t} \mid \widetilde{\mathfrak{M}}$, the set $\mathfrak{N} \cap \pi_{P}^{-1}(z)$ does not contain an interval, for any $z \in \mathfrak{M}$, and the lengths of the connected components of $\pi_{P}^{-1}(z) \backslash \mathfrak{N}$ are bounded below for $z \in \mathfrak{M}$; therefore, $\mathfrak{N} \cap \pi_{P}^{-1}(z)$ consists of finitely many points, and furthermore $\left.\pi_{P}\right|_{\mathfrak{N}}$ is a finite covering; we can also prove that an orbit disjoint from $\mathfrak{N}$ cannot approach $\mathfrak{N}$; therefore, $\psi_{t} \mid \widetilde{\mathfrak{M}}$ does not have exactly two minimal sets.)

We will restrict our attention to the case when $\psi_{t} \mid \widetilde{\mathfrak{M}}$ has exactly two minimal sets $\mathfrak{N}_{1}$ and $\mathfrak{N}_{2}$. In this case, we have the following properties of $\mathfrak{N}_{1}$ and $\mathfrak{N}_{2}$ from 
[Nakayama and Noda 2005], where $(S)_{z}$ denotes the intersection $S \cap \pi_{P}^{-1}(z)$ for a set $S$ in $P X$ and $z \in M$ :

Proposition 1.1. (a) Either $\left(\mathfrak{N}_{1}\right)_{z}$ or $\left(\mathfrak{N}_{2}\right)_{z}$ consists of a single point for any $z \in \mathfrak{M}$.

(b) There is a residual set in $\mathfrak{M}$ on which both $\left(\mathfrak{N}_{1}\right)_{z}$ and $\left(\mathfrak{N}_{2}\right)_{z}$ consist of a single point.

(c) For $i=1,2$, let $\xi_{i}^{+}(z)$ and $\xi_{i}^{-}(z)$ denote the maximum and minimum points of $\left(\mathfrak{N}_{i}\right)_{z}$ for $z \in \mathfrak{M}$, with respect to the orientation of the fiber $(P X)_{z}$. Here, these points are uniquely determined by $(a)$. We observe that $\xi_{i}^{+}(z)$ and $\xi_{i}^{-}(z)$ may not be continuous in $z$, but are semicontinuous; this implies that, if $\left(\mathfrak{N}_{i}\right)_{z}$ is a single point, then $z$ is a continuity point of $\xi_{i}^{+}$and $\xi_{i}^{-}$. If we set

$$
\begin{aligned}
& K_{i}=\left\{(z, s) \in P X \mid \xi_{i}^{-}(z) \leq s \leq \xi_{i}^{+}(z)\right\}, \\
& E_{1}=\left\{(z, s) \in P X \mid \xi_{1}^{+}(z)<s<\xi_{2}^{-}(z)\right\}, \\
& E_{2}=\left\{(z, s) \in P X \mid \xi_{2}^{+}(z)<s<\xi_{1}^{-}(z)\right\},
\end{aligned}
$$

then $K_{i}$ is a closed invariant set in $\tilde{\mathfrak{M}}$ and $E_{i}$ is an open invariant set in $\tilde{\mathfrak{M}}$.

(d) By (c), there are two (continuous) sections $h_{1}: \mathfrak{M} \rightarrow P X$ and $h_{2}: \mathfrak{M} \rightarrow P X$ such that $h_{1}(\mathfrak{M})$ and $h_{2}(\mathfrak{M})$ are contained in $E_{1}$ and $E_{2}$, respectively. Thus, $h_{1}(\mathfrak{M})$ and $h_{2}(\mathfrak{M})$ separate $\mathfrak{N}_{1}$ and $\mathfrak{N}_{2}$ in $P X$. In particular, $P X$ is a trivial bundle over $\mathfrak{M}$.

Here we remark that [Nakayama and Noda 2005, Theorem 1(2)] was proved under the stronger condition that $\varphi_{t}$ is minimal on the whole 3-manifold and is not topologically equivalent to an irrational flow of $T^{3}$. However, its proof is also valid for the restriction of $\varphi_{t}$ to the minimal set $\mathfrak{M}$, if $\left.\varphi_{t}\right|_{\mathfrak{M}}$ is not uniformly quasiconformal. Now, as above, if $\psi_{t} \mid \widetilde{\mathfrak{M}}$ has exactly two minimal sets, then $\left.\varphi_{t}\right|_{\mathfrak{M}}$ is not uniformly quasiconformal.

The dynamics of $\psi_{t} \mid \widetilde{\mathfrak{M}}$ are still very complicated, as indicated by an example of Johnson [1981] and our Example 1.4. Thus, we will apply the next result of Contreras [2002]:

Let $\pi: V \rightarrow B$ be a symplectic vector bundle over a compact set $B$. Let $\Psi_{t}$ be a flow on $V$ which is a bundle map that preserves the canonical symplectic structure on each fiber. The flow $\Psi_{t}$ induces a flow $\Phi_{t}$ on $B$ satisfying $\Phi_{t} \circ \pi=$ $\pi \circ \Psi_{t}$. Contreras [2002] showed that, if $\Psi_{t}$ is a weakly partially hyperbolic flow whose stable and unstable bundles have the same dimension, then the restricted flow $\left.\Psi_{t}\right|_{\pi^{-1}\left(\Omega\left(\Phi_{t}\right)\right)}$ is hyperbolic where $\Omega\left(\Phi_{t}\right)$ is a nonwandering set of $\Phi_{t}$.

We will apply his result to the renormalized linear Poincaré flow $v_{t}$ and extend his theorem under the stronger condition that $\mathfrak{M}$ is a minimal set instead of a nonwandering set $\Omega\left(\Phi_{t}\right)$ and that $\psi_{t} \mid \widetilde{\mathfrak{M}}_{\text {has }}$ exactly two minimal sets (the precise definitions will be given in the next sections). In Section 2, we give relations 
between the dynamics of the renormalized linear Poincare flow $v_{t}$ and the projective flow $\psi_{t}$. In Section 3, if $\left.\psi_{t}\right|_{E_{i}}$ has a fiberwise-divergent orbit, we find two types of orbits of $\psi_{t}$ in $E_{i}$, one that does not approach $\mathfrak{N}_{1}$ and the other that does not approach $\mathfrak{N}_{2}$. Here, the cross-ratio plays an important role. If all the orbits of $\psi_{t}$ are fiberwise convergent, then $\varphi_{t}$ has a dominated splitting over $\mathfrak{M}$, which will be proved in Section 4. Hence:

Theorem 1.2. Let $\mathfrak{M}$ be a minimal set of $\varphi_{t}$. If $\psi_{t} \mid \widetilde{\mathfrak{M}}$ has exactly two minimal sets $\mathfrak{N}_{1}$ and $\mathfrak{N}_{2}$, then either

(1) $\varphi_{t}$ has a dominated splitting over $\mathfrak{M}$; or

(2) for any $i=1,2$, there exist points $(z, s)$ and $\left(z^{\prime}, s^{\prime}\right)$ of $E_{i}$ such that

$$
\overline{O(z, s)} \cap \mathfrak{N}_{1}=\varnothing \quad \text { and } \quad \overline{O\left(z^{\prime}, s^{\prime}\right)} \cap \mathfrak{N}_{2}=\varnothing ;
$$

in particular, $\left.\psi_{t}\right|_{E_{i}}$ has an orbit which is not dense in $E_{i}$.

To take advantage of finding two types of orbits, we apply Theorem 1.2 to almost-periodic flows (defined in Section 5):

Theorem 1.3. If $\left.\varphi_{t}\right|_{\mathfrak{M}}$ is an almost-periodic minimal flow and $\psi_{t} \mid \widetilde{\mathfrak{M}}$ has exactly two minimal sets, then either

(1) $\varphi_{t}$ has a dominated splitting over $\mathfrak{M}$; or

(2) both $\left.\psi_{t}\right|_{E_{1}}$ and $\left.\psi_{t}\right|_{E_{2}}$ have dense orbits.

Finally, we give an example (communicated to the author by T. Noda) of an almost-periodic minimal flow on $T^{3}$ satisfying the conditions (2) of Theorems 1.2 and 1.3:

Example 1.4. Let $g: S^{1} \rightarrow S^{1}$ be a $C^{\omega}$ diffeomorphism such that $g$ is topologically conjugate to an irrational rotation $R_{\alpha}$ but is not $C^{1}$-conjugate to $R_{\alpha}$; such an example was constructed by Arnold [Herman 1979]. Let $\beta$ be an irrational number which is $\mathbb{Q}$-independent of $\alpha$. We define a $C^{\omega}$ diffeomorphism $f: T^{2} \rightarrow T^{2}$ by $f(x, y)=(g(x), y+\beta)$. Let $\varphi_{t}$ denote its suspension flow on $T^{3}$. Since $g$ is topologically conjugate to an irrational rotation, $\varphi_{t}$ is an almost-periodic minimal flow. On the other hand, $\sup _{n \geq 0}\left|\log D g^{n}(x)\right|=\infty$ for any $x \in S^{1}$, because $g$ is not $C^{1}$-conjugate to a rotation [Herman 1979]. Therefore, the projective flow of $\varphi_{t}$ has exactly two minimal sets, corresponding to the $x$-direction and the $y$-direction. Furthermore, by [Arroyo and Rodriguez Hertz 2003, Theorem B], we can show that $\varphi_{t}$ has no dominated splitting. Thus, the conditions (2) of Theorems 1.2 and 1.3 hold. The key point of this example is that such an example can be constructed as a $C^{\omega}$ flow. 


\section{Relations between lengths and angles}

Throughout this paper, we assume that $\mathfrak{M}$ is a minimal set of $\varphi_{t}$ and $\psi_{t} \mid \widetilde{\mathfrak{M}}$ has exactly two minimal sets $\mathfrak{N}_{1}$ and $\mathfrak{N}_{2}$, where $\tilde{\mathfrak{M}}=\pi_{P}^{-1}(\mathfrak{M})$. By (d) in Proposition 1.1, there exist two sections $h_{1}: \mathfrak{M} \rightarrow P X$ and $h_{2}: \mathfrak{M} \rightarrow P X$ separating $\mathfrak{N}_{1}$ and $\mathfrak{N}_{2}$. We choose a trivialization of $\tilde{\mathfrak{M}}=\mathfrak{M} \times \mathbb{P}^{1}$ so that

$$
h_{1}(\mathfrak{M})=\mathfrak{M} \times\{0\} \quad \text { and } \quad h_{2}(\mathfrak{M})=\mathfrak{M} \times\left\{\frac{\pi}{2}\right\},
$$

where the coordinate of $\mathbb{P}^{1}$ is given by $\left[-\frac{\pi}{2}, \frac{\pi}{2}\right] /-\frac{\pi}{2} \sim \frac{\pi}{2}$. Let $p_{2}: \tilde{\mathfrak{M}} \rightarrow \mathbb{P}^{1}$ denote the projection to the second factor.

In this section, we prepare several properties of orbits of $v_{t}$ and $\psi_{t}$, which will be frequently used throughout this paper.

We only consider the properties of $\psi_{t}$ restricted to

$$
E_{1}=\left\{(z, s) \in P X \mid \xi_{1}^{+}(z)<s<\xi_{2}^{-}(z)\right\} .
$$

Similar properties hold for $\left.\psi_{t}\right|_{E_{2}}$. We define

$$
\begin{array}{lll}
\Delta_{1}: E_{1} \rightarrow \mathbb{R} & \text { by } & \Delta_{1}(z, s)=s-\xi_{1}^{+}(z)>0, \\
\Delta_{2}: E_{1} \rightarrow \mathbb{R} & \text { by } & \Delta_{2}(z, s)=\xi_{2}^{-}(z)-s>0 .
\end{array}
$$

Let $e_{1}(z)$ and $e_{2}(z)$ denote the unit vectors of $\pi_{N}^{-1}(z)$ representing $\xi_{1}^{+}(z)$ and $\xi_{2}^{-}(z)$, respectively, where $e_{2}(z)$ is assumed to be on the positive side of $e_{1}(z)$. Set

$$
\Delta(z)=\xi_{2}^{-}(z)-\xi_{1}^{+}(z)
$$

Lemma 2.1. There is a real number $C>0$ such that, for any $z \in \mathfrak{M}$,

$$
\frac{1}{C} \leq\left|v_{t}\left(e_{1}(z)\right)\right|\left|v_{t}\left(e_{2}(z)\right)\right| \leq C
$$

Proof. Since $\xi_{2}^{-}\left(\varphi_{t}(z)\right)=\psi_{t}\left(\xi_{2}^{-}(z)\right)$ and $\xi_{1}^{+}\left(\varphi_{t}(z)\right)=\psi_{t}\left(\xi_{1}^{+}(z)\right)$, the angle between $v_{t}\left(e_{1}(z)\right)$ and $v_{t}\left(e_{2}(z)\right)$ is equal to $\Delta\left(\varphi_{t}(z)\right)$. Now, $v_{t}$ preserves the area of the triangle defined by the vectors $e_{1}(z)$ and $e_{2}(z)$. Hence, the area

$$
\left|v_{t}\left(e_{1}(z)\right)\right|\left|v_{t}\left(e_{2}(z)\right)\right| \sin \Delta\left(\varphi_{t}(z)\right)
$$

is invariant under $t \in \mathbb{R}$. Thus, we obtain

$$
\left|v_{t}\left(e_{1}(z)\right)\right|\left|v_{t}\left(e_{2}(z)\right)\right|=\left|\frac{\sin \Delta(z)}{\sin \Delta\left(\varphi_{t}(z)\right)}\right| .
$$

Since $\mathfrak{N}_{1}$ and $\mathfrak{N}_{2}$ are disjoint closed sets, we can choose $C>0$ (independent of $z \in \mathfrak{M})$ so that $1 / C \leq\left|\sin \Delta(z) / \sin \Delta\left(\varphi_{t}(z)\right)\right| \leq C$. 
Lemma 2.2. For $(z, s) \in E_{1}$, we have

$$
\inf _{t \geq 0} \frac{\left|v_{t}\left(e_{2}(z)\right)\right|}{\left|v_{t}\left(e_{1}(z)\right)\right|}=0
$$

if and only if $\inf _{t \geq 0} \Delta_{1}\left(\psi_{t}(z, s)\right)=0$. In particular, if $\inf _{t \geq 0} \Delta_{1}\left(\psi_{t}(z, s)\right)=0$ for some $(z, s) \in E_{1}$, then $\inf _{t \geq 0} \Delta_{1}\left(\psi_{t}\left(z, s^{\prime}\right)\right)=0$ for any $\left(z, s^{\prime}\right) \in E_{1}$. Similarly,

$$
\inf _{t \geq 0} \frac{\left|v_{t}\left(e_{1}(z)\right)\right|}{\left|v_{t}\left(e_{2}(z)\right)\right|}=0
$$

if and only if $\inf _{t \geq 0} \Delta_{2}\left(\psi_{t}(z, s)\right)=0$ for $(z, s) \in E_{1}$.

Proof. Let $v=k_{1} e_{1}(z)+k_{2} e_{2}(z)$ with $k_{1}, k_{2}>0$ denote the unit vector of $\pi_{N}^{-1}(z)$ representing $(z, s)$ of $E_{1}$. We denote by $\vartheta_{t}$ the angle between $v_{t}(v)$ and $v_{t}\left(e_{1}(z)\right)$. By the law of sines for the triangle defined by $v_{t}\left(k_{1} e_{1}(z)\right)$ and $v_{t}\left(k_{2} e_{2}(z)\right)$, we obtain

$$
\frac{\left|v_{t}\left(k_{1} e_{1}(z)\right)\right|}{\sin \left(\Delta\left(\varphi_{t}(z)\right)-\vartheta_{t}\right)}=\frac{\left|v_{t}\left(k_{2} e_{2}(z)\right)\right|}{\sin \vartheta_{t}}
$$

Thus,

$$
\frac{\left|v_{t}\left(e_{2}(z)\right)\right|}{\left|v_{t}\left(e_{1}(z)\right)\right|}=\frac{k_{1}}{k_{2}} \frac{\tan \vartheta_{t}}{\sin \Delta\left(\varphi_{t}(z)\right)-\cos \Delta\left(\varphi_{t}(z)\right) \tan \vartheta_{t}} .
$$

Since $\sin \Delta\left(\varphi_{t}(z)\right)$ is bounded below, $\inf _{t \geq 0}\left|v_{t}\left(e_{2}(z)\right)\right| /\left|v_{t}\left(e_{1}(z)\right)\right|=0$ if and only if $\inf _{t \geq 0} \vartheta_{t}=0$, which is equivalent to $\inf _{t \geq 0} \Delta_{1}\left(\psi_{t}(z, s)\right)=0$.

The next lemma is efficient for the study of the dynamical properties of $\left.\psi_{t}\right|_{E_{1}}$.

Lemma 2.3. For $(z, s) \in E_{1}$, we have $\inf _{t \geq 0} \Delta_{1}\left(\psi_{t}(z, s)\right)=0$ if and only if

$$
\overline{O_{+}(z, s)} \cap \mathfrak{N}_{1} \neq \varnothing .
$$

Proof. It is enough to show that $\overline{O_{+}(z, s)} \cap \mathfrak{N}_{1}=\varnothing$ if $\inf _{t \geq 0} \Delta_{1}\left(\psi_{t}(z, s)\right)$ is greater than 0.

Let $C=\min \left\{\inf _{t \geq 0} \Delta_{1}\left(\psi_{t}(z, s)\right), \pi / 2\right\}$. By (b) in Proposition 1.1, there is a point $z_{1}$ of $\mathfrak{M}$ such that $\mathfrak{N}_{1} \cap \pi_{P}^{-1}\left(z_{1}\right)$ consists of a single point. Then, $\xi_{1}^{ \pm}$is continuous at $z_{1}$. We choose a neighborhood $U_{1}$ of $z_{1}$ so that $\sup _{x \in U_{1}} \xi_{1}^{+}(x)-$ $\inf _{x \in U_{1}} \xi_{1}^{-}(x)<C$. For some small $\varepsilon>0$, let $W_{1}$ be the open set

$$
\left\{(x, u) \in E_{1} \mid x \in U_{1}, \inf _{y \in U_{1}} \xi_{1}^{-}(y)-\varepsilon<u<\inf _{y \in U_{1}} \xi_{1}^{-}(y)+C\right\} .
$$

Since $\Delta_{1}\left(\psi_{t}(z, s)\right) \geq C$ for $t \geq 0$, the point $\psi_{t}(z, s)$ is not contained in $W_{1}$ for $t \geq 0$. On the other hand, $\pi_{P}^{-1}\left(U_{1}\right) \cap \mathfrak{N}_{1}$ is contained in $W_{1}$, because, if $x \in U_{1}$, then $\xi_{1}^{+}(x) \leq \sup _{y \in U_{1}} \xi_{1}^{+}(y)<\inf _{y \in U_{1}} \xi_{1}^{-}(y)+C$. Therefore, $\bigcup_{t \leq 0} \psi_{t}\left(W_{1}\right)$ is a neighborhood of $\mathfrak{N}_{1}$ disjoint from $O_{+}(z, s)$, which implies $\overline{O_{+}(z, s)} \cap \mathfrak{N}_{1}=\varnothing$. 
Lemma 2.4. If $(z, s)$ is a point of $E_{1}$ satisfying

$$
\lim _{t \rightarrow+\infty} \Delta_{1}\left(\psi_{t}(z, s)\right)=0
$$

(respectively, $\left.\lim _{t \rightarrow-\infty} \Delta_{1}\left(\psi_{t}(z, s)\right)=0\right)$, then

$$
\lim _{t \rightarrow+\infty}\left|v_{t}(v)\right|=\infty
$$

(respectively, $\lim _{t \rightarrow-\infty}\left|v_{t}(v)\right|=\infty$ ), for any $v \in \pi_{N}^{-1}(z)-\{0\}$ with $[v] \in \mathfrak{N}_{1}$, where $[v]$ denotes the element of $P X$ represented by $v$.

Proof. Suppose that $\lim _{t \rightarrow+\infty} \Delta_{1}\left(\psi_{t}(z, s)\right)=0$. By the same proof as that of Lemma 2.2, $\left|v_{t}\left(e_{2}(z)\right)\right| /\left|v_{t}\left(e_{1}(z)\right)\right|$ converges to 0 as $t \rightarrow+\infty$. By Lemma 2.1, $\left|v_{t}\left(e_{1}(z)\right)\right|\left|v_{t}\left(e_{2}(z)\right)\right|$ is bounded below. Thus, $\left|v_{t}\left(e_{1}(z)\right)\right|$ tends toward $\infty$ as $t \rightarrow$ $+\infty$, because

$$
\left|v_{t}\left(e_{1}(z)\right)\right|^{2}=\frac{\left|v_{t}\left(e_{1}(z)\right)\right|}{\left|v_{t}\left(e_{2}(z)\right)\right|}\left|v_{t}\left(e_{1}(z)\right)\right|\left|v_{t}\left(e_{2}(z)\right)\right| .
$$

Again by Lemma 2.1, we obtain $\lim _{t \rightarrow+\infty}\left|v_{t}\left(e_{2}(z)\right)\right|=0$, and hence, for any $v \in \pi_{N}^{-1}(z)-\{0\}$ with $[v] \in \mathfrak{N}_{1}, \lim _{t \rightarrow+\infty}\left|v_{t}(v)\right|=\infty$.

The other case (that is, when $\lim _{t \rightarrow-\infty} \Delta_{1}\left(\psi_{t}(z, s)\right)=0$ ) can be shown in the same way.

\section{Fiberwise divergent orbits}

An orbit $O(z, s)$ of $\psi_{t}$ in $E_{i}(i=1,2)$ is called fiberwise convergent if either

$$
\lim _{t \rightarrow+\infty} \Delta_{1}\left(\psi_{t}(z, s)\right)=0 \quad \text { or } \quad \lim _{t \rightarrow+\infty} \Delta_{2}\left(\psi_{t}(z, s)\right)=0
$$

and either

$$
\lim _{t \rightarrow-\infty} \Delta_{1}\left(\psi_{t}(z, s)\right)=0 \quad \text { or } \quad \lim _{t \rightarrow-\infty} \Delta_{2}\left(\psi_{t}(z, s)\right)=0 .
$$

If not, we call it fiberwise divergent.

Lemma 3.1. If there is a point $\left(z_{1}, s_{1}\right)$ of $E_{i}(i=1,2)$ whose orbit is fiberwise divergent, then there exist points $\left(z_{2}, s_{2}\right)$ and $\left(z_{3}, s_{3}\right)$ of $E_{i}$ such that

$$
\overline{O\left(z_{2}, s_{2}\right)} \cap \mathfrak{N}_{1}=\varnothing \quad \text { and } \quad \overline{O\left(z_{3}, s_{3}\right)} \cap \mathfrak{N}_{2}=\varnothing .
$$

Proof. Let $O\left(z_{1}, s_{1}\right)$ be an orbit of $\psi_{t}$ in $E_{1}$ which is fiberwise divergent. We assume first that neither $\lim _{t \rightarrow+\infty} \Delta_{1}\left(\psi_{t}\left(z_{1}, s_{1}\right)\right)=0$ nor $\lim _{t \rightarrow+\infty} \Delta_{2}\left(\psi_{t}\left(z_{1}, s_{1}\right)\right)=$ 0 . Since $\omega\left(z_{1}, s_{1}\right)$ contains a minimal set, $\omega\left(z_{1}, s_{1}\right)$ contains $\mathfrak{N}_{1}$ or $\mathfrak{N}_{2}$. If $\omega\left(z_{1}, s_{1}\right)$ contains $\mathfrak{N}_{1}$, then $\inf _{t \geq 0} \Delta_{1}\left(\psi_{t}\left(z_{1}, s_{1}\right)\right)=0$ by Lemma 2.3 . Then, there exist points $\left(z_{2}, s_{2}\right)$ and $\left(z_{3}, s_{3}\right)$ of $E_{1}$ such that $\overline{O\left(z_{2}, s_{2}\right)} \cap \mathfrak{N}_{1}=\varnothing$ and $\overline{O\left(z_{3}, s_{3}\right)} \cap \mathfrak{N}_{2}=\varnothing$ by Lemma 3.3 at the end of this section. The other cases in Lemma 3.1 can be proved similarly. 
In order to later prove Lemma 3.3, we introduce the cross-ratio of straight lines in the plane: Let $s$ be an element of $\mathbb{P}^{1}$. We denote by $l$ the straight line in the plane, passing through the origin and representing $s$. If $\rho(s)$ (in $\mathbb{R} \cup\{\infty\}$ ) denotes the slope of $l$, then $(1, \rho(s)) \in \mathbb{R}^{2}$ is the intersection of $l$ and the line $\{(1, y) \mid y \in \mathbb{R}\}$. We give the coordinate of $\mathbb{P}^{1}$ by $\left[-\frac{\pi}{2}, \frac{\pi}{2}\right] / \sim$, and thus $\rho(s)=\tan s$.

Let $s_{1}, s_{2}, s_{3}$ and $s_{4}$ be elements of $\mathbb{P}^{1}$. The cross-ratio $\left(s_{1}, s_{2}, s_{3}, s_{4}\right)$ is

$$
\left(s_{1}, s_{2}, s_{3}, s_{4}\right)=\frac{\rho\left(s_{1}\right)-\rho\left(s_{3}\right)}{\rho\left(s_{3}\right)-\rho\left(s_{2}\right)} / \frac{\rho\left(s_{1}\right)-\rho\left(s_{4}\right)}{\rho\left(s_{4}\right)-\rho\left(s_{2}\right)} .
$$

It is invariant under the action of $\operatorname{PSL}(2 ; \mathbb{R})$. In particular, for $(z, s)$ and $\left(z, s^{\prime}\right)$ in $E_{1}$, the cross-ratio $\left(\xi_{1}^{+}\left(\varphi_{t}(z)\right), \xi_{2}^{-}\left(\varphi_{t}(z)\right), p_{2} \psi_{t}(z, s), p_{2} \psi_{t}\left(z, s^{\prime}\right)\right)$ is invariant under $t \in \mathbb{R}$. Now, we define $R_{1}, R_{2}: E_{1} \rightarrow \mathbb{R}$ by

$$
R_{1}(z, s)=\frac{\rho(s)-\rho\left(\xi_{1}^{+}(z)\right)}{\rho\left(\xi_{2}^{-}(z)\right)-\rho\left(\xi_{1}^{+}(z)\right)} \quad \text { and } \quad R_{2}(z, s)=\frac{\rho\left(\xi_{2}^{-}(z)\right)-\rho(s)}{\rho\left(\xi_{2}^{-}(z)\right)-\rho\left(\xi_{1}^{+}(z)\right)} .
$$

We have

$\left(\xi_{1}^{+}\left(\varphi_{t}(z)\right), \xi_{2}^{-}\left(\varphi_{t}(z)\right), p_{2} \psi_{t}(z, s), p_{2} \psi_{t}\left(z, s^{\prime}\right)\right)$

$$
=\frac{R_{1}\left(\psi_{t}(z, s)\right)}{1-R_{1}\left(\psi_{t}(z, s)\right)} / \frac{R_{1}\left(\psi_{t}\left(z, s^{\prime}\right)\right)}{1-R_{1}\left(\psi_{t}\left(z, s^{\prime}\right)\right)} .
$$

Lemma 3.2. If there exists a sequence $\left\{\left(z_{n}, s_{n}\right)\right\}_{n=1,2, \ldots}$ in $E_{1}$ such that

$$
R_{1}\left(\psi_{t}\left(z_{n}, s_{n}\right)\right) \geq R_{1}\left(z_{n}, s_{n}\right)
$$

for $-n \leq t \leq n$, then there is a $z_{\infty}$ in $\mathfrak{M}$ such that $\overline{O\left(z_{\infty}, 0\right)} \cap \mathfrak{N}_{1}=\varnothing$.

On the other hand, if there exists a sequence $\left\{\left(z_{n}^{\prime}, s_{n}^{\prime}\right)\right\}_{n=1,2, \ldots}$ in $E_{1}$ such that

$$
R_{2}\left(\psi_{t}\left(z_{n}^{\prime}, s_{n}^{\prime}\right)\right) \geq R_{2}\left(z_{n}^{\prime}, s_{n}^{\prime}\right)
$$

for $-n \leq t \leq n$, then there is a $z_{\infty}^{\prime}$ in $\mathfrak{M}$ such that $\overline{O\left(z_{\infty}^{\prime}, 0\right)} \cap \mathfrak{N}_{2}=\varnothing$.

Proof. Let $a_{t}=R_{1}\left(\psi_{t}\left(z_{n}, s_{n}\right)\right)$ and $b_{t}=R_{1}\left(\psi_{t}\left(z_{n}, 0\right)\right)$, where $\left(z_{n}, 0\right)$ is contained in the section $h_{1}(\mathfrak{M})$ by the choice of projective structure in Section 2. Since $h_{1}(\mathfrak{M})$ is contained in $E_{1}$, we have $0<a_{t}<1$ and $0<b_{t}<1$. By the invariance of the cross-ratios along orbits, we have

$$
\frac{a_{t}}{1-a_{t}} / \frac{b_{t}}{1-b_{t}}=\frac{a_{0}}{1-a_{0}} / \frac{b_{0}}{1-b_{0}} .
$$

Since $a_{t} \geq a_{0}$ for $t \in[-n, n]$, we obtain $b_{t} \geq b_{0}$. Hence,

$$
\frac{\rho\left(p_{2} \psi_{t}\left(z_{n}, 0\right)\right)-\rho\left(\xi_{1}^{+}\left(\varphi_{t}\left(z_{n}\right)\right)\right)}{\rho\left(\xi_{2}^{-}\left(\varphi_{t}\left(z_{n}\right)\right)\right)-\rho\left(\xi_{1}^{+}\left(\varphi_{t}\left(z_{n}\right)\right)\right)} \geq \frac{\rho(0)-\rho\left(\xi_{1}^{+}\left(z_{n}\right)\right)}{\rho\left(\xi_{2}^{-}\left(z_{n}\right)\right)-\rho\left(\xi_{1}^{+}\left(z_{n}\right)\right)} .
$$


Now, $\rho\left(\xi_{2}^{-}\right)-\rho\left(\xi_{1}^{+}\right)$is bounded above, because $\left(\mathfrak{N}_{1} \cup \mathfrak{N}_{2}\right) \cap h_{2}(\mathfrak{M})=\varnothing$. Since $\mathfrak{N}_{1}$ and $h_{1}(\mathfrak{M})$ are disjoint closed sets, $\rho(0)-\rho\left(\xi_{1}^{+}\left(z_{n}\right)\right)$ is bounded below. Thus, there exists $C_{2}>0$ such that $\Delta_{1}\left(\psi_{t}\left(z_{n}, 0\right)\right)>C_{2}$ for any $n$ and $t \in[-n, n]$.

Let $\left(z_{\infty}, 0\right)$ be an accumulating point of $\left\{\left(z_{n}, 0\right)\right\}$. Let $x$ be a point of $\mathfrak{M}$ on which $\left(\mathfrak{N}_{1}\right)_{x}$ is a single point. There is a neighborhood $W$ of $\left(x, \xi_{1}^{+}(x)\right)$ such that $\Delta_{1}(y, w)<C_{2}$ whenever $(y, w) \in W \cap E_{1}$.

We claim that $O\left(z_{\infty}, 0\right) \cap W=\varnothing$. Suppose there is a $t_{1}$ such that $\psi_{t_{1}}\left(z_{\infty}, 0\right) \in W$. If $n$ is a sufficiently large number satisfying $-n \leq t_{1} \leq n$, then $\Delta_{1}\left(\psi_{t_{1}}\left(z_{n}, 0\right)\right)>C_{2}$, and hence $\psi_{t_{1}}\left(z_{n}, 0\right)$ is not contained in $W$. However, this contradicts the continuity of $\psi_{t_{1}}$. Hence, $O\left(z_{\infty}, 0\right)$ is disjoint from $W$.

The invariant set $\bigcup_{t \in \mathbb{R}} \psi_{t}(W)$ is a neighborhood of $\mathfrak{N}_{1}$ disjoint from $O\left(z_{\infty}, 0\right)$. Therefore, $\overline{O\left(z_{\infty}, 0\right)} \cap \mathfrak{N}_{1}=\varnothing$.

The second part of the lemma can be proved in the same way as above, for $R_{2}=1-R_{1}$.

Lemma 3.3. If $\left(z_{1}, s_{1}\right)$ is a point of $E_{1}$ satisfying $\inf _{t \geq 0} \Delta_{1}\left(\psi_{t}\left(z_{1}, s_{1}\right)\right)=0$ but $\Delta_{1}\left(\psi_{t}\left(z_{1}, s_{1}\right)\right)$ does not converge to 0 as $t \rightarrow \infty$, then there exist points $\left(z_{2}, s_{2}\right)$ and $\left(z_{3}, s_{3}\right)$ of $E_{1}$ such that

$$
\overline{O\left(z_{2}, s_{2}\right)} \cap \mathfrak{N}_{1}=\varnothing \quad \text { and } \quad \overline{O\left(z_{3}, s_{3}\right)} \cap \mathfrak{N}_{2}=\varnothing .
$$

Proof. Since $R_{1}\left(\psi_{t}\left(z_{1}, s_{1}\right)\right)$ does not converge to 0 as $t \rightarrow \infty$, there are $C_{1}>0$ and $\left\{t_{n}\right\}_{n=1,2, \ldots}\left(t_{n} \geq 0\right)$ such that $\lim _{n \rightarrow \infty} t_{n}=\infty$ and $R_{1}\left(\psi_{t_{n}}\left(z_{1}, s_{1}\right)\right)>C_{1}$. Set

$$
\begin{aligned}
& K=\left\{(z, s) \in E_{1} \mid R_{1}(z, s) \geq C_{1}\right\}, \\
& W_{n}=E_{1} \backslash\left\{\psi_{t}(z, s) \mid(z, s) \in K,-n \leq t \leq n\right\} .
\end{aligned}
$$

Let $C_{2}=C_{1} \min _{z \in \mathfrak{M}}\left\{\rho\left(\xi_{2}^{-}(z)\right)-\rho\left(\xi_{1}^{+}(z)\right)\right\}$. Since the set

$$
\left\{\left(z, s, s^{\prime}, t\right) \in \mathfrak{M} \times \mathbb{P}^{1} \times \mathbb{P}^{1} \times \mathbb{R}|| \rho(s)-\rho\left(s^{\prime}\right) \mid \geq C_{2},-n \leq t \leq n\right\}
$$

is compact, the number

$$
\begin{aligned}
\varepsilon_{n}=\inf \left\{\frac{\left|\rho\left(p_{2} \psi_{t}(z, s)\right)-\rho\left(p_{2} \psi_{t}\left(z, s^{\prime}\right)\right)\right|}{\rho\left(\xi_{2}^{-}\left(\varphi_{t}(z)\right)\right)-\rho\left(\xi_{1}^{+}\left(\varphi_{t}(z)\right)\right)} \mid\right. \\
\left.(z, s),\left(z, s^{\prime}\right) \in \bar{E}_{1}, \quad-n \leq t \leq n,\left|\rho(s)-\rho\left(s^{\prime}\right)\right| \geq C_{2}\right\}
\end{aligned}
$$

is positive.

We denote by $V_{n}$ the set $\left\{(z, s) \in E_{1} \mid R_{1}(z, s)<\varepsilon_{n}\right\}$. We claim that $V_{n}$ is contained in $W_{n}$. Suppose that a point $(z, s)$ of $E_{1}$ is not contained in $W_{n}$. Then, there is a $t_{0} \in[-n, n]$ such that $\psi_{t_{0}}(z, s)$ is contained in $K$. Hence,

$$
\rho\left(p_{2} \psi_{t_{0}}(z, s)\right)-\rho\left(\xi_{1}^{+}\left(\varphi_{t_{0}}(z)\right)\right) \geq C_{1}\left(\rho\left(\xi_{2}^{-}\left(\varphi_{t_{0}}(z)\right)\right)-\rho\left(\xi_{1}^{+}\left(\varphi_{t_{0}}(z)\right)\right)\right) \geq C_{2} .
$$


The following inequality holds:

$$
R_{1}(z, s)=\frac{\rho\left(p_{2} \psi_{-t_{0}}\left(\varphi_{t_{0}}(z), p_{2} \psi_{t_{0}}(z, s)\right)\right)-\rho\left(p_{2} \psi_{-t_{0}}\left(\varphi_{t_{0}}(z), \xi_{1}^{+}\left(\varphi_{t_{0}}(z)\right)\right)\right)}{\rho\left(\xi_{2}^{-}\left(\varphi_{-t_{0}}\left(\varphi_{t_{0}}(z)\right)\right)\right)-\rho\left(\xi_{1}^{+}\left(\varphi_{-t_{0}}\left(\varphi_{t_{0}}(z)\right)\right)\right)} \geq \varepsilon_{n} .
$$

Thus, $(z, s)$ is not a point of $V_{n}$. This implies that $V_{n} \subset W_{n}$.

By the assumption that inf $\operatorname{t\geq 0}_{1} \Delta_{1}\left(\psi_{t}\left(z_{1}, s_{1}\right)\right)=0$, we have

$$
\inf _{t \geq 0} R_{1}\left(\psi_{t}\left(z_{1}, s_{1}\right)\right)=0 .
$$

We choose an increasing sequence $\left\{v_{n}\right\}_{n=1,2, \ldots}$ in $\mathbb{R}$ so that $\psi_{v_{2 n-1}}\left(z_{1}, s_{1}\right) \in K$ and $\psi_{v_{2 n}}\left(z_{1}, s_{1}\right) \in V_{n}$. Let $u_{n}$ be the time between $v_{2 n-1}$ and $v_{2 n+1}$ when $R_{1}$ is minimum at $\psi_{u_{n}}(z, s)$, that is,

$$
R_{1}\left(\psi_{u_{n}}\left(z_{1}, s_{1}\right)\right)=\min \left\{R_{1}\left(\psi_{t}\left(z_{1}, s_{1}\right)\right) \mid v_{2 n-1} \leq t \leq v_{2 n+1}\right\} .
$$

Then, $R_{1}\left(\psi_{u_{n}}\left(z_{1}, s_{1}\right)\right)$ is smaller than $\varepsilon_{n}$, because $R_{1}\left(\psi_{v_{2 n}}\left(z_{1}, s_{1}\right)\right)<\varepsilon_{n}$. Hence, $\psi_{u_{n}}\left(z_{1}, s_{1}\right)$ is contained in $V_{n}$ and thus in $W_{n}$. Since we have $\psi_{u_{n}}\left(z_{1}, s_{1}\right) \in W_{n}$ and $\psi_{v_{2 n-1}}\left(z_{1}, s_{1}\right) \in K$, the time difference $u_{n}-v_{2 n-1}$ is greater than $n$. Furthermore, we obtain $v_{2 n+1}-u_{n}>n$, because $\psi_{u_{n}}\left(z_{1}, s_{1}\right) \in W_{n}$ and $\psi_{v_{2 n+1}}\left(z_{1}, s_{1}\right) \in K$.

By the above construction, if $-n \leq t \leq n$, the following inequalities hold:

$$
v_{2 n-1}<u_{n}-n \leq t+u_{n} \leq u_{n}+n<v_{2 n+1}, \quad R_{1}\left(\psi_{t+u_{n}}\left(z_{1}, s_{1}\right)\right) \geq R_{1}\left(\psi_{u_{n}}\left(z_{1}, s_{1}\right)\right) .
$$

Therefore, $\left\{\psi_{u_{n}}\left(z_{1}, s_{1}\right)\right\}_{n=1,2, \ldots}$ satisfies the first condition of Lemma 3.2, and hence there is a point $z_{2}$ of $\mathfrak{M}$ such that $\overline{O\left(z_{2}, 0\right)} \cap \mathfrak{N}_{1}=\varnothing$.

Let $w_{n}$ be the time between $v_{2 n}$ and $v_{2 n+2}$ when $R_{2}$ is minimum, that is,

$$
R_{2}\left(\psi_{w_{n}}\left(z_{1}, s_{1}\right)\right)=\min \left\{R_{2}\left(\psi_{t}\left(z_{1}, s_{1}\right)\right) \mid v_{2 n} \leq t \leq v_{2 n+2}\right\} .
$$

Since $R_{1}=1-R_{2}, \quad \psi_{w_{n}}\left(z_{1}, s_{1}\right)$ is contained in $K$. Hence, $w_{n}-v_{2 n} \geq n$ and $v_{2 n+2}-w_{n} \geq n$. Thus, $R_{2}\left(\psi_{t+w_{n}}\left(z_{1}, s_{1}\right)\right) \geq R_{2}\left(\psi_{w_{n}}\left(z_{1}, s_{1}\right)\right)$ for $-n \leq t \leq n$. Therefore, $\left\{\psi_{w_{n}}\left(z_{1}, s_{1}\right)\right\}_{n=1,2, \ldots}$ satisfies the second condition of Lemma 3.2. As a consequence, there exists a point $z_{3}$ of $\mathfrak{M}$ such that $\overline{O\left(z_{3}, 0\right)} \cap \mathfrak{N}_{2}=\varnothing$.

\section{Fiberwise-convergent orbits}

Let $\Lambda$ be a closed $\varphi_{t}$-invariant set in $M$. A $C^{1}$ flow $\varphi_{t}$ admits a dominated splitting over $\Lambda$ if there is a continuous splitting of $\pi_{N}^{-1}(\Lambda)$ into a direct sum of 1dimensional bundles $S$ and $U$, invariant under $\tilde{D} \varphi_{t}$, such that there are constants $C>0$ and $\lambda \in(0,1)$ satisfying

$$
\frac{\left\|\left.\tilde{D} \varphi_{t}\right|_{S(z)}\right\|}{\left\|\left.\tilde{D} \varphi_{t}\right|_{U(z)}\right\|} \leq C \lambda^{t}
$$

for any $z$ and $t \geq 0$, where $\tilde{D} \varphi_{t}$ is the map on $N X=T M / T \varphi_{t}$ induced from $D \varphi_{t}$, 
while $\|\cdot\|$ denotes the operator norm from some continuous Riemannian metric. The above inequality is equivalent to

$$
\frac{\left\|\left.v_{t}\right|_{S(z)}\right\|}{\left\|\left.v_{t}\right|_{U(z)}\right\|} \leq C \lambda^{t} .
$$

We consider the case when $\Lambda$ is a minimal set. For example, if $\Lambda$ is a hyperbolic closed orbit, then $\varphi_{t}$ admits a dominated splitting over $\Lambda$. On the other hand, a minimal $C^{2}$ flow on a closed 3-manifold does not admit a dominated splitting over the whole 3-manifold, as was proved by Arroyo and Rodriguez Hertz [2003].

In this section, we will consider the case when all the orbits of $\psi_{t}$ contained in $E_{1}$ are fiberwise convergent. The orbit of $\psi_{t}$ passing through $(z, s) \in E_{1}$ is then classified into four types:

(I) $\lim _{t \rightarrow+\infty} \Delta_{1}\left(\psi_{t}(z, s)\right)=0$ and $\lim _{t \rightarrow-\infty} \Delta_{2}\left(\psi_{t}(z, s)\right)=0$;

(II) $\lim _{t \rightarrow+\infty} \Delta_{2}\left(\psi_{t}(z, s)\right)=0$ and $\lim _{t \rightarrow-\infty} \Delta_{1}\left(\psi_{t}(z, s)\right)=0$;

(III) $\lim _{t \rightarrow+\infty} \Delta_{1}\left(\psi_{t}(z, s)\right)=0$ and $\lim _{t \rightarrow-\infty} \Delta_{1}\left(\psi_{t}(z, s)\right)=0$;

(IV) $\lim _{t \rightarrow+\infty} \Delta_{2}\left(\psi_{t}(z, s)\right)=0$ and $\lim _{t \rightarrow-\infty} \Delta_{2}\left(\psi_{t}(z, s)\right)=0$.

To prove Theorem 1.2, we will investigate the case when there is no orbit of type IV. The following lemma of Contreras and Iturriaga [1999] plays an important role; we change their hypothesis to suit our purpose, but the proof is the same.

Lemma 4.1 [Contreras and Iturriaga 1999, Lemma 3.3]. If $\sup _{t \in \mathbb{R}}\left|v_{t}(v)\right|=\infty$ for any $v \neq 0$ with $[v] \in \mathfrak{N}_{1}$, then there is a $C_{1}>0$ such that

$$
\left|v_{t}\left(e_{1}(z)\right)\right| \leq C_{1}\left(1+\left|v_{s}\left(e_{1}(z)\right)\right|\right)
$$

for any $z, s$ and $t$ with $0 \leq t \leq s$.

Lemma 4.2. If all the orbits of $\psi_{t}$ in $E_{1}$ are of type I, II or III, then all the orbits are of type I or all the orbits are of type II.

Proof. It is enough to show that, if all the orbits of $\psi_{t}$ are of type I, II or III and there is an orbit of type I or III, then all the orbits are of type I.

By the assumption that there is no orbit of type IV, we have

$$
\lim _{t \rightarrow+\infty} \Delta_{1}\left(\psi_{t}(z, s)\right)=0 \quad \text { or } \quad \lim _{t \rightarrow-\infty} \Delta_{1}\left(\psi_{t}(z, s)\right)=0
$$

for any $(z, s) \in E_{1}$. By Lemma 2.4, $\sup _{t \in \mathbb{R}}\left|v_{t}(v)\right|=\infty$ for any $v \neq 0$ with $[v] \in \mathfrak{N}_{1}$. Thus, by Lemma 4.1, there is a $C_{1}>0$ such that $\left|v_{t}\left(e_{1}(z)\right)\right| \leq C_{1}\left(1+\left|v_{s}\left(e_{1}(z)\right)\right|\right)$ for any $z, s$ and $t$ with $0 \leq t \leq s$. If $\left(z_{1}, s_{1}\right)$ is a point of type I or III, then $\lim _{t \rightarrow+\infty} \Delta_{1}\left(\psi_{t}\left(z_{1}, s_{1}\right)\right)=0$, and hence $\lim _{t \rightarrow+\infty}\left|v_{t}\left(e_{1}\left(z_{1}\right)\right)\right|=\infty$ by Lemma 2.4 .

Let $z_{2}$ be an arbitrary point of $\mathfrak{M}$. Since $\mathfrak{M}$ is a minimal set, there is a sequence $\left\{t_{n}\right\}_{n=1,2, \ldots}$ of positive numbers such that $\lim _{n \rightarrow+\infty} t_{n}=\infty$ and $\varphi_{t_{n}}\left(z_{1}\right) \rightarrow z_{2}$. 
Taking a subsequence of $\left\{t_{n}\right\}$, we can assume that $v_{t_{n}}\left(e_{1}\left(z_{1}\right)\right) /\left|v_{t_{n}}\left(e_{1}\left(z_{1}\right)\right)\right|$ converges to some unit vector $v_{2}$. Then $\left[v_{2}\right]$ is contained in $\left(\mathfrak{N}_{1}\right)_{z_{2}}$. Since we have $\lim _{t \rightarrow+\infty}\left|v_{t}\left(e_{1}\left(z_{1}\right)\right)\right|=\infty$, the constant $C_{2}=\inf _{t \geq 0}\left|v_{t}\left(e_{1}\left(z_{1}\right)\right)\right|$ is positive. For $0 \leq t \leq t_{n}$, we have

$$
\left|v_{-t}\left(\frac{v_{t_{n}}\left(e_{1}\left(z_{1}\right)\right)}{\left|v_{t_{n}}\left(e_{1}\left(z_{1}\right)\right)\right|}\right)\right|=\frac{\left|v_{t_{n}-t}\left(e_{1}\left(z_{1}\right)\right)\right|}{\left|v_{t_{n}}\left(e_{1}\left(z_{1}\right)\right)\right|} \leq \frac{C_{1}\left(1+\left|v_{t_{n}}\left(e_{1}\left(z_{1}\right)\right)\right|\right)}{\left|v_{t_{n}}\left(e_{1}\left(z_{1}\right)\right)\right|} \leq C_{1}\left(1+\frac{1}{C_{2}}\right) .
$$

Therefore, $\left|v_{-t}\left(v_{2}\right)\right|$ is bounded above for $t \geq 0$. By Lemma 2.4, for $\left(z_{2}, s\right) \in E_{1}$, $\Delta_{1}\left(\psi_{t}\left(z_{2}, s\right)\right)$ does not converge to 0 as $t \rightarrow-\infty$. Since all the orbits are of type I, II or III, the orbit passing through $\left(z_{2}, s\right)$ is of type I.

Proof of Theorem 1.2. We prove that, if $\varphi_{t}$ has no dominated splitting over $\mathfrak{M}$, there exist points $(z, s)$ and $\left(z^{\prime}, s^{\prime}\right)$ of $E_{1}$ with $\overline{O(z, s)} \cap \mathfrak{N}_{1}=\varnothing$ and $\overline{O\left(z^{\prime}, s^{\prime}\right)} \cap \mathfrak{N}_{2}=\varnothing$. The proof for $\left.\psi_{t}\right|_{E_{2}}$ is the same. By Lemma 3.1, we can further assume that all the orbits of $\psi_{t}$ in $E_{1}$ are fiberwise convergent.

If there is no orbit of type IV, then, by Lemma 4.2, all the orbits are of type I or all the orbits are of type II. But then $\varphi_{t}$ has a dominated splitting over $\mathfrak{M}$ by a standard argument (for example, $\left.\varphi_{t}\right|_{\mathfrak{M}}$ is weakly partially hyperbolic [Contreras 2002]). Therefore, there exists an orbit of type IV. We can show in the same way that there exists an orbit of type III. Thus, there exist points $(z, s)$ and $\left(z^{\prime}, s^{\prime}\right)$ of $E_{1}$ such that $\overline{O(z, s)} \cap \mathfrak{N}_{1}=\varnothing$ and $\overline{O\left(z^{\prime}, s^{\prime}\right)} \cap \mathfrak{N}_{2}=\varnothing$.

\section{Almost-periodic minimal flows}

We prove Theorem 1.3. A subset $A$ of $\mathbb{R}$ is called syndetic if $\mathbb{R}=\{a+k \mid a \in$ $A, k \in K\}$ for some compact set $K$ of $\mathbb{R}$. A flow $\varphi_{t}$ on a compact metric space $\mathfrak{M}$ is called almost periodic if, for any $\varepsilon>0$, there is a syndetic set $A$ such that $d\left(z, \varphi_{a}(z)\right)<\varepsilon$ for any $z \in \mathfrak{M}$ and $a \in A$, where $d$ is a metric of $\mathfrak{M}$. If the whole $\mathfrak{M}$ is a minimal set, then the flow is called minimal.

Almost-periodic minimal flows on a compact metric space $\mathfrak{M}$ are already classified in the topological sense. In fact, they are equivalent to equicontinuous minimal flows, see [Auslander 1988, Theorem, p. 36]. Furthermore, there are invariant metrics on $\mathfrak{M}$, and $\mathfrak{M}$ is a compact abelian group, see [Auslander 1988, Exercises, p. 45]. However, the infinitesimal behavior of almost-periodic minimal flows is still complicated, as Example 1.4 indicates (see also [Nakayama 2001]).

With Theorem 1.2, if $\varphi_{t}$ has no dominated splitting, we find two types of orbits. Thus, we deduce Theorem 1.3 by using the next lemma:

Lemma 5.1. Assume that $\left.\varphi_{t}\right|_{\mathfrak{M}}$ is an almost-periodic minimal flow and $\psi_{t} \mid \widetilde{\mathfrak{M}}$ has exactly two minimal sets. If, for some $i \in\{1,2\}$, we have both:

(1) $\psi_{t}$ has a positive semiorbit in $E_{i}$ that does not approach $\mathfrak{N}_{1}$ (that is, $\overline{O_{+}(z, s)}$ and $\mathfrak{N}_{1}$ are disjoint for some $\left.(z, s)\right)$, and 
(2) $\psi_{t}$ has a positive semiorbit in $E_{i}$ that does not approach $\mathfrak{N}_{2}$ (that is, $\overline{O_{+}(z, s)}$ and $\mathfrak{N}_{2}$ are disjoint for some $\left.(z, s)\right)$;

then $\left.\psi_{t}\right|_{E_{i}}$ has a dense orbit.

Proof. We only prove this lemma for $\left.\psi_{t}\right|_{E_{1}}$.

Let $W_{1}$ and $W_{2}$ be arbitrary open sets in $E_{1}$. We only have to show that

$$
\left(\bigcup_{t \in \mathbb{R}} \psi_{t}\left(W_{2}\right)\right) \cap W_{1} \neq \varnothing .
$$

We choose open sets $U_{1}$ and $U_{2}$ in $\mathfrak{M}$ and open intervals $I_{1}=\left(\alpha_{1}, \beta_{1}\right)$ and $I_{2}=$ $\left(\alpha_{2}, \beta_{2}\right)$, so that $\overline{U_{1} \times I_{1}} \subset W_{1}$ and $\overline{U_{2} \times I_{2}} \subset W_{2}$. Then it is enough to show that $\left(\bigcup_{t \in \mathbb{R}} \psi_{t}\left(U_{2} \times I_{2}\right)\right) \cap\left(U_{1} \times I_{1}\right) \neq \varnothing$.

First, we claim that there are a connected open set $V_{2}$ contained in $U_{2}$ and a syndetic set $A$, such that $\varphi_{a}\left(V_{2}\right)$ is contained in $U_{1}$ for any $a \in A$. Let $z_{3}$ be a point of $U_{1}$. There is an $\varepsilon>0$ such that the $\varepsilon$-ball $B_{\varepsilon}\left(z_{3}\right)$ with center $z_{3}$ is contained in $U_{1}$. By the minimality of $\varphi_{t}$, there is a $t_{1} \in \mathbb{R}$ such that $\varphi_{t_{1}}\left(z_{3}\right)$ is contained in $U_{2}$. Let $V_{2}$ be a connected component of $U_{2} \cap \varphi_{t_{1}}\left(B_{\varepsilon / 2}\left(z_{3}\right)\right)$. For any $y \in V_{2}$, we have $d\left(\varphi_{-t_{1}}(y), z_{3}\right)<\varepsilon / 2$. Since $\varphi_{t}$ is almost periodic, there is a syndetic set $A^{\prime}$ such that $d\left(\varphi_{a}(x), x\right)<\varepsilon / 2$ for any $x \in \mathfrak{M}$ and $a \in A^{\prime}$. In particular, $d\left(\varphi_{a-t_{1}}(y), \varphi_{-t_{1}}(y)\right)<\varepsilon / 2$ for any $a \in A^{\prime}$. Hence, $d\left(\varphi_{a-t_{1}}(y), z_{3}\right)<\varepsilon$, which implies that $\varphi_{a-t_{1}}(y)$ is contained in $U_{1}$. Since $\left\{a-t_{1} \mid a \in A^{\prime}\right\}$ is also syndetic, the claim follows. Hence, for any $t \in \mathbb{R}$, there is a $u \in\left[0, C_{1}\right]$ satisfying $\varphi_{t+u}\left(V_{2}\right) \subset U_{1}$.

The set

$$
\left\{z \in \mathfrak{M} \mid \overline{O_{+}(z, s)} \cap \mathfrak{N}_{1}=\varnothing \text { for any }(z, s) \in E_{1}\right\}
$$

is a nonempty invariant set in $\mathfrak{M}$, by Lemmas 2.2 and 2.3. Hence, it is dense in $\mathfrak{M}$, because $\left.\varphi_{t}\right|_{\mathfrak{M}}$ is minimal. Furthermore, the set

$$
\left\{z \mid \overline{O_{+}(z, s)} \cap \mathfrak{N}_{2}=\varnothing \text { for any }(z, s) \in E_{1}\right\}
$$

is also dense in $\mathfrak{M}$. Thus, there are points $\left(z_{1}, s_{1}\right)$ and $\left(z_{2}, s_{2}\right)$ of $V_{2} \times I_{2}$ such that $\overline{O_{+}\left(z_{1}, s_{1}\right)} \cap \mathfrak{N}_{1}=\varnothing$ and $\overline{O_{+}\left(z_{2}, s_{2}\right)} \cap \mathfrak{N}_{2}=\varnothing$. Since $\psi_{t}$ contains no minimal set in $E_{1}$, we have $\overline{O_{+}\left(z_{1}, s_{1}\right)} \cap \mathfrak{N}_{2} \neq \varnothing$ and $\overline{O_{+}\left(z_{2}, s_{2}\right)} \cap \mathfrak{N}_{1} \neq \varnothing$.

Set

$$
\begin{aligned}
& F_{2}=\left\{(z, s) \in E_{1} \mid z \in \bar{U}_{1}, s \leq \beta_{1}\right\}, \\
& W_{2}=E_{1} \backslash\left\{\psi_{t}(z, s) \mid(z, s) \in F_{2},-C_{1} \leq t \leq 0\right\} .
\end{aligned}
$$

Then, $W_{2} \cup K_{2}$ is a neighborhood of $\mathfrak{N}_{2}$ in $E_{1} \cup K_{2}$, where

$$
K_{i}=\left\{(z, s) \in P X \mid \xi_{i}^{-}(z) \leq s \leq \xi_{i}^{+}(z)\right\},
$$

as in Section 1. If $(z, s) \in W_{2}$, then, for $t \in\left[0, C_{1}\right], \psi_{t}(z, s)$ is not contained in $F_{2}$. If we further assume that $\varphi_{t}(z) \in U_{1}$, then $p_{2} \psi_{t}(z, s)>\beta_{1}$.

We claim that there exists a $C_{2}>0$ such that, for any $t \geq 0$, there is a $u \in\left[0, C_{2}\right]$ such that $\psi_{t+u}\left(z_{1}, s_{1}\right) \in W_{2}$. If not, there is a sequence $\left\{t_{n}^{\prime}\right\}_{n=1,2, \ldots}$ with $t_{n}^{\prime} \geq 0$, such 
that $\psi_{t_{n}^{\prime}+u}\left(z_{1}, s_{1}\right) \notin W_{2}$ for $0 \leq u \leq n$. Let $\left(z_{0}, s_{0}\right)$ be an accumulating point of $\left\{\psi_{t_{n}^{\prime}}\left(z_{1}, s_{1}\right)\right\}_{n=1,2, \ldots}$. The positive semiorbit starting from $\left(z_{0}, s_{0}\right)$ is disjoint from $W_{2}$. Thus, $\overline{O_{+}\left(z_{0}, s_{0}\right)} \cap \mathfrak{N}_{2}=\varnothing$. On the other hand, $O_{+}\left(z_{1}, s_{1}\right)$ is disjoint from a neighborhood of $\mathfrak{N}_{1}$. Hence, $\psi_{t_{n}^{\prime}+u}\left(z_{1}, s_{1}\right)$ is disjoint from this neighborhood for $0 \leq u \leq n$. Thus, $\overline{O_{+}\left(z_{0}, s_{0}\right)} \cap \mathfrak{N}_{1}=\varnothing$. Therefore, the $\omega$-limit set of $\left(z_{0}, s_{0}\right)$ contains a minimal set different from $\mathfrak{N}_{1}$ and $\mathfrak{N}_{2}$, which contradicts the assumption.

We construct a neighborhood $W_{1}$ of $\mathfrak{N}_{1}$, similarly to the construction of $W_{2}$ as a neighborhood of $\mathfrak{N}_{2}$, by using the constant $C_{1}+C_{2}$. Set

$$
\begin{aligned}
& F_{1}=\left\{(z, s) \in E_{1} \mid z \in \bar{U}_{1}, s \geq \alpha_{1}\right\} \\
& W_{1}=E_{1} \backslash\left\{\psi_{t}(z, s) \mid(z, s) \in F_{1},-\left(C_{1}+C_{2}\right) \leq t \leq 0\right\} .
\end{aligned}
$$

Then, $W_{1} \cup K_{1}$ is a neighborhood of $\mathfrak{N}_{1}$ in $E_{1} \cup K_{1}$ such that, if $(z, s) \in W_{1}$ and $\varphi_{t}(z) \in U_{1}$ for some $0 \leq t \leq C_{1}+C_{2}$, then $p_{2} \psi_{t}(z, s)<\alpha_{1}$.

We will choose $t$ (as $\left.t=t_{2}+t_{3}+t_{4}\right)$ so that $\psi_{t}\left(U_{2} \times I_{2}\right) \cap\left(U_{1} \times I_{1}\right) \neq \varnothing$. First, we choose $t_{2} \geq 0$ so that $\psi_{t_{2}}\left(z_{2}, s_{2}\right) \in W_{1}$. By the choice of $C_{2}$, there is a $t_{3} \in\left[0, C_{2}\right]$ such that $\psi_{t_{2}+t_{3}}\left(z_{1}, s_{1}\right) \in W_{2}$. Finally, we take $t_{4} \in\left[0, C_{1}\right]$ so that $\varphi_{t_{2}+t_{3}+t_{4}}\left(V_{2}\right)$ is contained in $U_{1}$. Since $\psi_{t_{2}}\left(z_{2}, s_{2}\right) \in W_{1}$ and $\varphi_{t_{2}+t_{3}+t_{4}}\left(z_{2}\right) \in U_{1}$, we have $p_{2} \psi_{t_{2}+t_{3}+t_{4}}\left(z_{2}, s_{2}\right)<\alpha_{1}$. On the other hand, $p_{2} \psi_{t_{2}+t_{3}+t_{4}}\left(z_{1}, s_{1}\right)>\beta_{1}$, because $\psi_{t_{2}+t_{3}}\left(z_{1}, s_{1}\right) \in W_{2}$ and $\varphi_{t_{2}+t_{3}+t_{4}}\left(z_{1}\right) \in U_{1}$. Therefore, $\psi_{t_{2}+t_{3}+t_{4}}$ maps an arc contained in $V_{2} \times I_{2}$ that joins $\left(z_{1}, s_{1}\right)$ and $\left(z_{2}, s_{2}\right)$ onto an arc contained in $\pi_{P}^{-1}\left(U_{1}\right)$ which intersects $U_{1} \times I_{1}$. Thus, $\psi_{t_{2}+t_{3}+t_{4}}\left(V_{2} \times I_{2}\right) \cap\left(U_{1} \times I_{1}\right) \neq \varnothing$.

Now, at the end of the paper, we comment on the "cocycle condition" for the projective flows with exactly two minimal sets $\mathfrak{N}_{1}$ and $\mathfrak{N}_{2}$ : Assume that $\mathfrak{N}_{1}$ and $\mathfrak{N}_{2}$ are the image of two (continuous) sections, and change the trivialization of $P X=\mathfrak{M} \times \mathbb{P}^{1}=\mathfrak{M} \times\left[-\frac{\pi}{2}, \frac{\pi}{2}\right] / \sim$ so that $\mathfrak{N}_{1}=\mathfrak{M} \times\{0\}$ and $\mathfrak{N}_{2}=\mathfrak{M} \times\left\{\frac{\pi}{2}\right\}$. Then, for $z \in \mathfrak{M}$, the restriction of $\psi_{t}$ to the fiber can be written

where

$$
\left(\begin{array}{cc}
1 & 0 \\
0 & a_{t}(z)
\end{array}\right)
$$

$$
\left(\begin{array}{cc}
1 & 0 \\
0 & a_{t}(z)
\end{array}\right)\left(\begin{array}{c}
1 \\
\rho(s)
\end{array}\right)=\left(\begin{array}{c}
1 \\
\rho\left(s^{\prime}\right)
\end{array}\right)
$$

if $\psi_{t}(z, s)=\left(\varphi_{t}(z), s^{\prime}\right)$. Thus, $a_{t_{1}+t_{2}}(z)=a_{t_{2}}\left(\varphi_{t_{1}}(z)\right) a_{t_{1}}(z)$ for $t_{1}, t_{2} \in \mathbb{R}$. Now, $\psi_{t}$ has exactly two minimal sets, $\mathfrak{N}_{1}$ and $\mathfrak{N}_{2}$. Thus, $\left|\sum_{i=0}^{n} \log a_{1}\left(\varphi_{i}(z)\right)\right|=\left|\log a_{n}(z)\right|$ is not bounded. By Gottschalk and Hedlund [1955, Theorem 14.11], there is no continuous function $h: \mathfrak{M} \rightarrow \mathbb{R}$ such that $h\left(\varphi_{1}(z)\right)-h(z)=\log a_{1}(z)$ if $\varphi_{1}$ is minimal. 


\section{References}

[Arroyo and Rodriguez Hertz 2003] A. Arroyo and F. Rodriguez Hertz, "Homoclinic bifurcations and uniform hyperbolicity for three-dimensional flows", Ann. Inst. H. Poincaré Anal. Non Linéaire 20:5 (2003), 805-841. MR 2004j:37047 Zbl 1045.37006

[Auslander 1988] J. Auslander, Minimal flows and their extensions, North-Holland Mathematics Studies 153, North-Holland, Amsterdam, 1988. MR 89m:54050 Zbl 0654.54027

[Brunella 1996] M. Brunella, “On transversely holomorphic flows, I", Invent. Math. 126:2 (1996), 265-279. MR 97j:58121 Zbl 0873.57021

[Contreras 2002] G. Contreras, "Partially hyperbolic geodesic flows are Anosov", C. R. Math. Acad. Sci. Paris 334:7 (2002), 585-590. MR 2003e:37036 Zbl 01754822

[Contreras and Iturriaga 1999] G. Contreras and R. Iturriaga, "Convex Hamiltonians without conjugate points”, Ergodic Theory and Dynamical Systems 19:4 (1999), 901-952. MR 2000h:37102 Zbl 1044.37046

[Ghys 1996] É. Ghys, "On transversely holomorphic flows, II", Invent. Math. 126:2 (1996), 281286. MR 97j:58122 Zbl 0873.57022

[Gottschalk and Hedlund 1955] W. H. Gottschalk and G. A. Hedlund, Topological dynamics, AMS Colloquium Publications 36, American Mathematical Society, Providence, R. I., 1955. MR 17,650e Zbl 0067.15204

[Herman 1979] M.-R. Herman, "Sur la conjugaison différentiable des difféomorphismes du cercle à des rotations", Inst. Hautes Études Sci. Publ. Math. 49 (1979), 5-233. MR 81h:58039 Zbl 0448.58019

[Johnson 1981] R. A. Johnson, "A linear, almost periodic equation with an almost automorphic solution", Proc. Amer. Math. Soc. 82:2 (1981), 199-205. MR 82i:34044a Zbl 0474.34039

[Matsumoto and Nakayama 1997] S. Matsumoto and H. Nakayama, "On v-distal flows on 3-manifolds”, Bull. London Math. Soc. 29:5 (1997), 609-616. MR 98d:58156 Zbl 1047.37501

[Nakayama 2001] H. Nakayama, "Transitively twisted flows of 3-manifolds", Comment. Math. Helv. 76:4 (2001), 577-588. MR 2002m:37028 Zbl 0996.37017

[Nakayama and Noda 2005] H. Nakayama and T. Noda, "Minimal sets and chain recurrent sets of projective flows induced from minimal flows on 3-manifolds", Discrete Contin. Dyn. Syst. 12:4 (2005), 629-638. MR 2006f:37062 Zbl 1075.37011

[Sullivan 1981] D. Sullivan, "On the ergodic theory at infinity of an arbitrary discrete group of hyperbolic motions", pp. 465-496 in Riemann surfaces and related topics (Stony Brook, NY, 1978), edited by I. Kra and B. Maskit, Ann. of Math. Stud. 97, Princeton Univ. Press, Princeton, N.J., 1981. MR 83f:58052 Zbl 0567.58015

Received June 2, 2005. Revised August 27, 2005.

HIROMICHI NAKAYAMA

FACULTY OF INTEGRATED ARTS AND SCIENCES

HIROSHIMA UNIVERSITY

1-7-1 KAGAMIYAMA

HIGASHI-HIROSHIMA 739-8521

JAPAN

nakayama@mis.hiroshima-u.ac.jp 